\title{
Gestión del talento humano en salud pública. Un análisis en cinco ciudades colombianas, $2014^{*}$
}

\section{Talent Management in Public Health; an Analysis of Five Colombian Cities, 2014}

\section{Gestão do talento humano em saúde pública. Análise em cinco cidades colombianas, 2014}

Fecha de recepción: 02-10-15 Fecha de aceptación: 08-03-16 Disponible en línea: 03-05-16 doi:10.11144/Javeriana.rgyps15-30.gtsp

Cómo citar este artículo:

Molina-Marím G, Oquendo-Lozano T, Rodríguez-Garzón S, Montoya-Gómez N, Vesga-Gómez C, LagosCampos N, Almanza-Payares R, Chavarro-Medina M, Goenaga-Jiménez E, Arboleda-Posada G. Gestión del talento humano en salud pública. Un análisis en cinco ciudades colombianas, 2014. Rev. Gerenc. Polít. Salud. 2016; 15(30): 108-125. http://dx.doi.org/10.11144/Javeriana.rgyps15-30.gtsp

\author{
Gloria Molina-Marím** \\ Tatiana Oquendo-Lozano*** \\ Sergio Rodríguez-Garzón**** \\ Nilton Montoya-Gómez***** \\ Celmira Vesga-Gómez $* * * * * *$ \\ Nancy Lagos-Campos $* * * * * * *$ \\ Rita Almanza-Payares $* * * * * * * *$ \\ Martha Chavarro-Medina********* \\ Eloina Goenaga-Jiménez $* * * * * * * * *$ \\ Gladys Arboleda-Posada***********
}

Este artículo es uno de los productos de la investigación titulada Decisiones en la política y la gestión en la salud pública en el ámbito municipal. Un análisis en seis ciudades colombianas en el contexto del sistema general de seguridad social en salud, 2012-2014, financiada por Colciencias (contrato no 420-2011), Universidad de Antioquia, con la participación de la Universidad Nacional de Colombia, la Universidad Santo Tomas y la Universidad de Illinois - Chicago.

** Enfermera, Ph. D. Docente de la Facultad Nacional de Salud Pública, Universidad de Antioquia. Correo electrónico: gloria.molina@udea.edu.co. Calle 62 No. 52-59, oficina 309, Medellín, Colombia.

**** Administradora en Salud, Facultad Nacional de Salud Pública, Universidad de Antioquia.

***** Gerente en Sistemas de Información en Salud, Facultad Nacional de Salud Pública, Universidad de Antioquia.

****** Estadístico, docente de la Facultad Nacional de Salud Pública, Universidad de Antioquia.

****** Enfermera, magíster en Salud Pública, Universidad Industrial de Santander.

******** Enfermera, magíster en Salud Pública. Secretaría de Salud de Pasto.

********* Enfermera, magíster en Salud Pública. Secretaría de Salud de Medellín.

********* Enfermera, Secretaría de Salud Departamental de Amazonas.

*********** Médica, Secretaría de Salud de Barranquilla.

************ Administradora de empresas, docente de la Facultad Nacional de Salud Pública, Universidad de Antioquia. 


\section{Resumen}

Objetivo: describir las condiciones laborales del personal de salud vinculado a programas de salud pública. Metodología: se realizó un estudio descriptivo de corte transversal a una muestra representativa de 672 personas vinculadas a instituciones de salud habilitadas por el Ministerio de Salud en el 2013, que proveían servicios y programas de salud pública. Resultados: los hallazgos sugieren que la mayor parte del personal vinculado a servicios y programas de salud pública tenía formación en medicina, enfermería y odontología, condiciones laborales problemáticas - dado que predomina la contratación de corto plazo- y la cobertura en capacitación en salud pública era baja. La mayor idoneidad en salud pública de los encuestados se asoció con haber aprobado el examen de conocimiento para ingresar a la institución y tener evaluación del desempeño. Conclusión: se evidenció limitación en la formación y en la capacitación y deficientes condiciones laborales del personal que laboraba en los programas de salud pública.

Palabras clave: recursos humanos en salud, política de salud, salud pública, competencias en salud pública

\section{Abstract}

Objective: describing the work conditions of the health personnel associated with public health programs. Methodology: we carried out a descriptive cross-section study on a representative sample consisting of 672 people associated with health institutions approved by the Ministry of Health in 2013, which provide public health programs and services. Results: findings suggest most of the personnel associated with public health services and programs had medicine, nursing, and dentistry training; had problematic working conditions - as short-term contracts are prevailing - and the public health training coverage was low. The most common form of adequacy in public health of the people surveyed was associated with approving the knowledge test necessary to become associated with the institution and with the performance assessments. Conclusions: it is possible to see limitations on training and education, as well as deficient working conditions for people who work in public health programs.

Keywords: human resources in health; health policies; public health; public health competencies

\section{Resumo}

Objetivo: descrever as condições laborais dos profissionais de saúde vinculados a programas de saúde pública. Metodologia: foi realizado um estudo descritivo de corte transversal em amostra representativa de 672 pessoas vinculadas a instituições de saúde habilitadas pelo Ministério de Saúde no 2013, provindo serviços e programas de saúde pública. Resultados: os achados sugerem que a maior parte do pessoal vinculado a serviços e programas de saúde pública tinha formação em medicina, enfermagem e odontologia, condições laborais problemáticas — dado que predomina a contratação de curto prazo - e a cobertura em capacitação em saúde pública é baixa. A maior idoneidade em saúde pública dos inquiridos foi associada com ter aprovado o teste de conhecimento para ingressar à instituição e ter avaliação de desempenho. Conclusão: evidenciou-se limitação na formação e treinamento e condições laborais deficientes do pessoal que laborava nos programas de saúde pública.

Palavras-chave: recursos humanos em saúde; política de saúde; saúde pública; competências em saúde pública 
Gloria Molina-Marím • Tatiana Oquendo-Lozano • Sergio Rodriguez-Garzón • Nilton Montoya-

Gómez • Celmira Vesga-Gómez • Nancy Lagos-Campos • Rita Almanza-Payares • Martha

Chavarro-Medina • Eloina Goenaga-Jiménez • Gladys Arboleda-Posada

\section{Introducción}

El talento humano constituye un recurso esencial en las instituciones de salud (1), por ello su adecuada gestión es crucial para el óptimo desempeño y el logro de las metas de dichas instituciones y del sistema de salud en su conjunto (2).

La Reunión Panamericana de Ministros de Salud, realizada en Toronto, Canadá en el 2005, se centró en los recursos humanos en salud. Allí se aprobó la iniciativa titulada "Llamado a la acción de Toronto para una década de recursos humanos en salud" (3), en la cual se establece: "los recursos humanos son la base del sistema de salud. La contribución de los trabajadores de la salud es un factor esencial de la mejoría de la calidad de vida; ellos son protagonistas de su desarrollo" (4). La oms define el talento humano en salud como "Todos los trabajadores remunerados empleados en organizaciones o instituciones cuya principal finalidad es mejorar la salud y [...] todos aquellos cuyas actividades individuales persiguen mayormente ese mismo objetivo pero que trabajan para otro tipo de organizaciones" (5). En Colombia, la Ley 1164 del 2007 define el talento humano en salud como "todo el personal que interviene en la promoción, educación, información de la salud, prevención, diagnóstico, tratamiento, rehabilitación y paliación de la enfermedad de los habitantes del territorio nacional, dentro de la estructura organizacional de la prestación de los servicios de salud" (6).

Dado el reconocido papel del talento humano en el logro de los objetivos de las instituciones y organizaciones (7), su gestión tiene importancia en la teoría y práctica administrativa, la cual incluye cinco procesos básicos (8): a) integración: mercado de recursos humanos, reclutamiento y selección; b) organización: diseño, descripción y análisis de puestos de trabajo y evaluación de desempeño; c) retención: remuneración y retribuciones, prestaciones y servicios sociales, higiene y seguridad en el trabajo y las relaciones sindicales; d) desarrollo: capacitación y desarrollo organizacional; e) evaluación: sistemas de información, controles, constancia, productividad y equilibrio social. Estos procesos están interconectados, cualquier cambio en uno de ellos afecta a los demás, y se relacionan con factores internos de la organización, ambientales y tecnológicos, entre otros. La Organización Panamericana de la Salud (OPS) entiende la gestión de recursos humanos como "el conjunto de procesos y actividades que ponen en funcionamiento, desarrollan y movilizan a los trabajadores de las organizaciones de salud para alcanzar los objetivos sanitarios establecidos. [...]. Se precisan métodos e instrumentos para conseguir, conservar y desarrollar los recursos humanos en salud que se reconocen como el valor más importante de toda organización de salud" (9).

Según Nigenda et al. (1), la contratación establece la relación entre el trabajador y la organización y determina las condiciones laborales. Las formas de contratación predominantes en el continente americano son: a) empleado público vinculado a una institución estatal, en la cual se han visto reducidas las garantías y prestaciones, debido a las políticas de flexibilidad laboral y con ello el crecimiento de la informalidad en las últimas décadas; b) contratación por cooperativas, que genera problemas de fragmentación de la fuerza laboral, tercerización o subcontratación de servicios; c) contratos temporales, por los cuales el trabajador, mediante una remuneración determinada, se obliga a prestar servicios a personas naturales o jurídicas, lo que no incluye prestaciones sociales ni estabilidad laboral.

Las condiciones laborales del talento humano también se vinculan con las características de las instituciones educativas encargadas de la 
formación, y con las dinámicas de oferta y demanda de servicios (1); los modelos de desarrollo y la riqueza de los países (9), y con los entornos geográficos, culturales, sociales, el orden público y la normatividad de cada país.

Colombia tiene reglamentación sobre personal de salud desde 1975 con el Decreto 056, en 1977 el Decreto 2905, en 1992 el Decreto 1849, en el 2002 la Ley 789 y en el 2007 la Ley 1164 de Talento Humano en Salud. Esta última ley define que "la formación y el desempeño del Talento Humano en Salud, debe caracterizarse por el logro de los mayores beneficios posibles en la formación y la atención [...]"; también relaciona "el ejercicio idóneo de competencias propias de cada profesión u ocupación en salud con la satisfacción y mejoramiento de la salud de los usuarios de los servicios". La Ley 1438 del 2011, que reforma el Sistema General de Seguridad Social en Salud (sGsss), establece que "el Ministerio de la Protección Social, [...] definirá la política de Talento Humano en Salud que oriente la formación, ejercicio y gestión de las profesiones y ocupaciones del área de la salud, en coherencia con las necesidades de la población colombiana, las características y objetivos del sGsss". La legislación colombiana reconoce la incidencia fundamental de los recursos humanos en el desarrollo de las estrategias en salud y su respectivo cumplimiento de objetivos. Sin embargo, en la práctica las condiciones laborales que se ofrecen en las instituciones de salud al parecer no dan cuenta de lo que la legislación establece.

El sGsss de Colombia —Ley 100 de 1993-se fundamenta en directrices internacionales encaminadas a mejorar la cobertura, el acceso, la calidad, la equidad, la eficiencia y la sostenibilidad financiera de los sistemas de salud. Esta ley estableció el aseguramiento como medio para acceder a los servicios, creó las Empresas Promotoras de Salud
(EPS) públicas y privadas, responsables del aseguramiento y de proveer un Plan Obligatorio de Salud (POS) a sus afiliados, por lo cual reciben una Unidad de Pago por Capitación (UPC) por persona afiliada. Esta ley también cambió el subsidio de la oferta por el subsidio de la demanda, introdujo el mercado y la competencia entre las EPS y las Instituciones Prestadoras de Servicios (IPS) públicas y privadas, redujo el papel del Estado y amplió la participación privada (10). A pesar de lo anterior, no se ha logrado un mayor acceso efectivo a los servicios de salud y a los programas de salud pública, debido a múltiples barreras administrativas, geográficas y económicas generadas, que tienen su origen en el interés de lucro financiero, principalmente por las EPS $(11,12)$. Además, se ha reportado que algunas EPS tienen asimetría de información, segmentan y seleccionan la población con menos riesgos en salud, provocan conflictos interinstitucionales por no pagar oportunamente a las IPS los servicios prestados y hacen pactos para negar servicios a los afiliados (13).

Este artículo se centra en presentar los resultados de un estudio realizado sobre talento humano vinculado a acciones en salud pública, cuyo objetivo fue analizar las características sociodemográficas y las condiciones laborales de este personal en el contexto del sGsss.

\section{Metodología}

Se realizó un estudio descriptivo de corte transversal (14) en el año 2014, en las siguientes cinco ciudades colombianas: Barranquilla, Bucaramanga, Leticia, Medellín y Pasto, en la cuales se había realizado un estudio cualitativo sobre los procesos de 
Gloria Molina-Marím • Tatiana Oquendo-Lozano • Sergio Rodriguez-Garzón • Nilton Montoya-

Gómez • Celmira Vesga-Gómez • Nancy Lagos-Campos • Rita Almanza-Payares • Martha

Chavarro-Medina • Eloina Goenaga-Jiménez • Gladys Arboleda-Posada

toma de decisiones en salud pública, ${ }^{1}$ del cual se derivó la necesidad de analizar las condiciones laborales, el clima organizacional y la idoneidad del talento humano vinculado al desarrollo de acciones en los programas de salud pública.

\section{Población, muestra y muestreo}

Se tomó la base de datos del Ministerio de Salud y Protección Social sobre IPs habilitadas que ofrecían programas de salud pública en las cinco ciudades en el 2013; se verificó telefónicamente la existencia de los programas registrados en cada una. Se identificaron 514 programas de Salud Pública en las IPS registradas en la base de datos. Para cada ciudad se realizó un muestreo aleatorio de instituciones con probabilidad proporcional al tamaño, es decir, al número de programas o servicios de salud pública que ofrecían (15). Entre más programas ofrecían, se asumió que la institución tenía más personal contratado para desarrollarlos. A cada programa de cada institución se le asignó un número entero diferente entre 1 y 514 . Luego se generaron $n$ números aleatorios entre 1 y 514 que siguen una distribución uniforme. Cuando uno de esos números coincidió con un programa $x$, la institución que lo ofrecía fue seleccionada para formar parte de la muestra. Las IPS con más de seis programas fueron incluidas obligatoriamente. Como resultado del proceso de muestreo antes descrito, el número de IPS seleccionadas de cada ciudad fue: once en Barranquilla, diez en Bucaramanga, siete en Leticia, diez en Medellín y doce en Pasto.

1 Los resultados de este estudio de tipo cualitativo están publicados en el capítulo 8- "Características de la gestión del talento humano en salud pública"del libro titulado Decisiones en la política y la gestión e salud pública. El bien común en confrontación con los intereses y prácticas particulares. Medellín: Pulso y Letra Editores; 2013. p. 265-95.
A cada una de las instituciones seleccionadas se le solicitó la información del personal que realizaba actividades de salud pública, la cual fue debidamente depurada. Con ello se construyó una base de datos de personal de cada una de las instituciones seleccionadas y se configuró como marco muestral por ciudad, el cual fue de 2860 personas. Para el cálculo de la muestra se aplicó la siguiente formula y criterios:

$$
n=\frac{Z_{1-\alpha / 2}^{2} P(1-P)}{e^{2}} ; \quad n^{\prime}=\frac{N n}{N+n-1}
$$

$\mathrm{N}$ : 2680, error relativo (E): 0,09; error de muestreo (e): 0,09, proporción (P): 0,30; $\alpha: 0,05$.

La muestra estimada fue de 562 personas más una sobremuestra del $20 \%$, con el fin de prever las pérdidas de unidades muestrales. La muestra total fue de 675 personas y se distribuyó proporcionalmente por ciudad e institución (tabla 1). Las personas encuestadas fueron elegidas mediante un proceso de selección aleatoria simple de la base de datos de cada institución previamente seleccionada en cada ciudad.

Se elaboró un cuestionario autodiligenciable, cuyo contenido incluye variables sociodemográficas (edad, sexo), formación académica, años de experiencia, tipo de contrato(s) laboral(es) en los últimos tres años (2012, 2013 y 2014), criterios de selección para el puesto de trabajo, salario promedio mensual, vinculación a la seguridad social, capacitación, idoneidad en salud pública, tipo y frecuencia de la evaluación del desempeño, incentivos, motivaciones y variables de clima organizacional, entre otros.

Este cuestionario se diseñó en el aplicativo E-encuesta, para dar respuesta on-line, para lo cual se envió mensaje por correo electrónico a las personas incluidas en la muestra. 
Tabla 1. Distribución de la MUESTRa de InStituciones y PERSONAS POR CIUdAd

\begin{tabular}{|l|c|l|c|c|}
\hline Ciudad & $\begin{array}{c}\text { Número } \\
\text { de IPS }\end{array}$ & $\begin{array}{l}\text { Número de personas contratadas en } \\
\text { programas de salud pública }\end{array}$ & $\%$ & Muestra (n) \\
\hline Pasto & 12 & 133 & 11,7 & 79 \\
\hline Bucaramanga & 10 & 190 & 16,7 & 113 \\
\hline Leticia & 7 & 218 & 19,2 & 129 \\
\hline Barranquilla & 11 & 191 & 16,8 & 113 \\
\hline Medellín & 10 & 405 & 35,6 & 240 \\
\hline Total & $\mathbf{5 0}$ & $\mathbf{1 1 3 7}$ & $\mathbf{1 0 0 , 0}$ & $\mathbf{6 7 5}$ \\
\hline
\end{tabular}

Fuente: elaboración propia

En el mensaje enviado se les suministró la información acerca del estudio y el consentimiento informado y se les invitó a contestar el cuestionario bajo su consentimiento. También se aplicó el mismo cuestionario cara a cara a las personas que por diferentes motivos no lograron responderlo on-line.

Se elaboró una base de datos con la información recolectada y se realizó depuración y control de calidad de los datos. Luego se procedió a realizar análisis descriptivo y con base en estos resultados se hicieron pruebas de asociación entre algunas variables.

\section{Limitaciones del estudio}

Imprecisión o desactualización de la información de personal de algunas instituciones, dada la alta rotación del personal ligado a los tipos de contratación; por tanto, algunas personas que estaban incluidas en la muestra inicial en una institución, ya no tenían contrato en esta y fue necesario buscarlas en otra institución o reemplazarlas.

Hubo dificultad en la recolección on-line, debido a limitaciones de acceso a internet (institucional o en casa), así como escasa capacidad para el manejo de las tecnologías digitales.

\section{Resultados}

Características sociodemográficas y formación académica de los encuestados

De las 675 personas seleccionadas en la muestra, respondieron 672 (99,5\%). 71,6\% de ellas es de sexo femenino, $26,8 \%$ de sexo masculino, y 1,6\% sin dato. El promedio de edad es de 39 años, la persona de menos edad tiene 20 años y la de más edad tiene 72 años.

El estado civil de los 672 encuestados es: casado(a) el 41,7\% (280 personas), soltero(a) el $33 \%$ (222 personas), unión libre el 13,7\% (92 personas), divorciado(a) 5,4\% (36 personas) viudo(a) el $0,9 \%$ (6 personas) y sin dato $5,4 \%$ (36 personas).

En cuanto a la formación académica, el $17,3 \%$ (116 personas) es auxiliar, el 18,8\% (126 personas) técnico/tecnólogo y el 65,8\% (442 personas) profesional. En el nivel auxiliar son en su mayoría auxiliares de enfermería y odontología. En los técnicos y tecnólogos predominan enfermería, salud oral, saneamiento y salud pública. En los profesionales predominan medicina, enfermería, odontología, psicología, bacteriología, trabajo social y nutrición y dietética (tabla 2). 
Gloria Molina-Marím • Tatiana Oquendo-Lozano • Sergio Rodriguez-Garzón • Nilton Montoya-

Gómez • Celmira Vesga-Gómez • Nancy Lagos-Campos • Rita Almanza-Payares • Martha

Chavarro-Medina • Eloina Goenaga-Jiménez • Gladys Arboleda-Posada

Tabla 2. Nivel aCAdÉMico de los EnCUESTAdos de las CINCO CIUDAdES

\begin{tabular}{|l|c|c|}
\hline Título de auxiliar & Frec. & $\%$ \\
\hline Auxiliar de enfermería & 89 & 78,1 \\
\hline Auxiliar de odontología & 10 & 8,8 \\
\hline $\begin{array}{l}\text { Bachiller (pedagógico, comer- } \\
\text { cio, otro) }\end{array}$ & 4 & 3,5 \\
\hline Auxiliar en salud pública & 3 & 2,6 \\
\hline Auxiliar de saneamiento & 2 & 1,8 \\
\hline Auxiliar en áreas de la salud & 2 & 1,8 \\
\hline Auxiliar de laboratorio clínico & 1 & 0,9 \\
\hline Auxiliar en gerontología & 1 & 0,9 \\
\hline $\begin{array}{l}\text { Auxiliar en vigilancia y control } \\
\text { en los factores de riesgos del } \\
\text { consumo }\end{array}$ & 1 & 0,9 \\
\hline Otros auxiliar & 1 & 0,9 \\
\hline Total & 114 & 100,0 \\
\hline Título de tecnólogo/técnicos & Frec. & $\%$ \\
\hline $\begin{array}{l}\text { Técnico profesional en enfer- } \\
\text { mería }\end{array}$ & 48 & 37,5 \\
\hline Técnico en saneamiento & 16 & 12,5 \\
\hline $\begin{array}{l}\text { Técnico profesional en salud } \\
\text { oral }\end{array}$ & 13 & 10,2 \\
\hline $\begin{array}{l}\text { Técnico profesional en salud } \\
\text { pública }\end{array}$ & 12 & 9,4 \\
\hline $\begin{array}{l}\text { Técnico administrativo: mer- } \\
\text { cadotecnia, comercial, costos, } \\
\text { auditoria, contabilidad }\end{array}$ & 6 & 4,7 \\
\hline Tecnólogo en sistemas & 5 & 3,9 \\
\hline Otros técnicos (1) & 128 & 100,0 \\
\hline Total & & \\
\hline
\end{tabular}

\begin{tabular}{|l|c|c|}
\hline Título de profesional & Frec. & $\%$ \\
\hline Médico & 184 & 41,7 \\
\hline Enfermera & 139 & 31,5 \\
\hline Odontólogo & 30 & 6,8 \\
\hline Psicólogo & 22 & 5,0 \\
\hline Bacteriólogo & 15 & 3,4 \\
\hline Trabajo social & 7 & 1,6 \\
\hline Nutricionista y dietista & 6 & 1,4 \\
\hline Fonoaudiólogo & 5 & 1,1 \\
\hline Terapeuta ocupacional & 3 & 0,7 \\
& & \\
\hline Abogado & 2 & 0,5 \\
\hline Biólogo & 2 & 0,5 \\
\hline Comunicador social & 2 & 0,5 \\
\hline Fisioterapeuta & 2 & 0,5 \\
\hline Gerente de la salud pública & 2 & 0,5 \\
\hline Ingeniero de sistemas & 2 & 0,5 \\
\hline Microbiólogo & 2 & 0,5 \\
\hline Optómetra & 2 & 0,5 \\
\hline Veterinario zootecnista & & \\
\hline Otros (2) & 2 & 0,5 \\
\hline Total & & 2,7 \\
\hline
\end{tabular}

(1) Gestión de empresas asociativas y organización comunitaria, normalista superior, técnico auxiliar de laboratorios, química industrial, atención y formación a la primera infancia, salud ambiental, gestión de los recursos naturales y del ambiente, promoción de la salud, alimentos, salud ocupacional, criminalística, vigilancia y control, regencia de farmacia.

(2) Administrador de empresas, contador público, ingeniería de alimentos, ingeniero electrónico, ingeniero químico, instrumentador quirúrgico, profesional administración, profesional en deporte y actividad física, profesional en salud ocupacional, sociólogo y terapeuta respiratoria.

Fuente: elaboración propia

En cuanto a la formación de posgrados, el 24,6\% (165 personas) tiene diplomado, el 20,5\% (138 114 personas) especialización, el 3,7\% (25 personas) maestría y el $0,3 \%$ (dos personas) doctorado.
De las veinticinco personas que cursaron maestría, el 24\% la realizó en salud pública, el $16 \%$ en educación, docencia universitaria o administración y supervisión educativa, el $8 \%$ 
en terapia familiar y el $8 \%$ en ciencias sociales. El 54\% restante realizó maestría en una de las siguientes áreas: administración en salud, ciencias básicas biomédicas, coordinación de trasplantes, desarrollo empresarial y gestión social, educación y desarrollo comunitario con énfasis en investigación cualitativa, literatura, problemas sociales contemporáneos, emergencias y desastres, salud sexual y reproductiva y teología.

Las 138 personas con especialización incluyen una amplia gama: $29 \%$ gerencia y administración en servicios de salud o temas afines, $21,7 \%$ en auditoría en servicios de salud, $15,2 \%$ salud ocupacional y $19,4 \%$ epidemiología. El 24,6\% restante realizó alguna de las siguientes especializaciones: conservación de recursos naturales, coordinación de trasplantes, cuidado del niño en estado crítico, desarrollo humano y educación sexual, dirección y gestión deportiva, docencia universitaria, enfermería maternoperinatal, laboratorio de hematología y banco de sangre, medicina familiar, medicina forense, medicina alternativa, nutrición animal sostenible, planeación para la educación ambiental, promoción y comunicación en salud, psicología clínica, psiquiatría de enlace, farmacodependencia o valoración en el daño de la salud mental, salud familiar y salud pública con énfasis en salud indígena.

Igualmente, los 164 profesionales que realizaron diplomados lo hicieron en alguna de las siguientes áreas:

- Especialidad clínica el 32,9\%: intervención terapéutica estética, estética dental, reanimación cerebro-cardiopulmonar básica y avanzada, urgencias y emergencias, diabetes, terapia cognitiva, discapacidad, materno-infantil, UCi neonatal, cuidados críticos, tratamiento contra el dolor, banco de sangre y/o actualización en medicina (complementaria, familiar, integrativa, homeopática, homotoxicología, tradicional china) y medicina general y/o urgencias.

- Promoción y educación en salud y prevención de la enfermedad el 29,9\%: nutrición en actividad física, en medicina deportiva o infantil, salud sexual y reproductiva, vacunación, víctimas de violencia sexual, salud ocupacional, docencia universitaria, Atención Primaria en Salud, programas de infancia como AIEPI y salud mental.

- Administración, gestión y evaluación el 26,8\%: plan de atención básica, salud ocupacional, mercadeo, manejo social de las organizaciones solidarias, políticas públicas, calidad, proyectos en salud pública, seguridad social, servicios de salud, recursos humanos, habilitación, acreditación, auditoría, control interno, garantía de la calidad en salud, HSEQ, guías de práctica clínica basadas en evidencia y/o Ley 100.

- Otras áreas el 10,4\%: derechos humanos, justicia transicional y posconflicto, humanización de servicios de salud, epidemiología clínica, estudio de las sagradas escrituras, proyectos en pueblos indígenas, redes de computadores o próxima generación y salud pública.

De los 184 médicos, 0,5\% tenía doctorado, $4,3 \%$ maestría y $23,9 \%$ especialización. De los 139 profesionales en enfermería, el 3,6\% tenía maestría, el 25,2\% especialización y el $27,3 \%$ estudios de diplomado. De los treinta odontólogos que respondieron la encuesta, el 43,3\% tenía especialización y el $40 \%$ diplomado. De los veintidós psicólogos, el 4,5\% (uno) tenía doctorado, 13,6\% maestría, $45,5 \%$ especialización y $45,5 \%$ diplomado. 


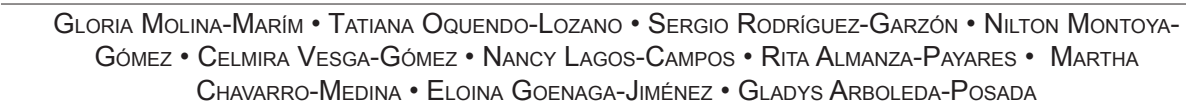

\section{Condiciones laborales}

Tipo de vinculación o contratación. De los 672 encuestados, el $40,2 \%$ en el 2012, el $48,4 \%$ en el 2013 y el 64,6\% en el 2014 afirmaron haber tenido o tener contrato relacionado con salud pública. La tabla 3 muestra el tipo de contrato, donde se observa que el 46,9\% (2014), el 45,9\% (2013) y el $51,9 \%$ (2012) tenía un contrato de carrera administrativa o indefinido, lo que implica mayor estabilidad. Entre tanto, el 53,0\% (2014), el 54,1\% (2013) y el 48,1\% (2012) tenían contrato por honorarios, comisiones, outsourcing, provisional o prestación de servicios, lo cual no garantiza continuidad y conlleva alta rotación del personal. En cuanto a la duración de los contratos en meses, se observa que las modalidades de contratación por servicios, comisiones, término fijo y outsourcing son las de menos duración.

Del total (672) de encuestados, el 7,9\% reportó haber estado trabajando alguna vez sin contrato durante los últimos años (2014, 2013 y 2012); de estas, el 19,2\% trabajó sin contrato por un mes, el $21,1 \%$ entre dos y tres meses, el 13,5\% durante seis meses y el $13,5 \%$ por doce meses.

El 82\% (552) de los encuestados respondió la pregunta relacionada con el tiempo que tardó en conseguir el trabajo que tenía en el momento de la encuesta. De estas personas, al 49,3\% le tomó hasta un mes, al 25,2\% entre dos y cinco meses, al $11,2 \%$ entre seis y nueve meses, al 7,4\% entre diez y catorce meses, y al 6,9\% entre 15 y 120 meses. La media es de cinco meses.

El 84,7\% (569 personas) informó en cuántas instituciones trabaja simultáneamente en el año 2014; el 16,0\% contestó trabajar en dos instituciones a la vezy el 2,5\% en tres instituciones. Esto significa que aproximadamente una de cada cinco personas trabajaba en dos o más instituciones simultáneamente.

El 81,2\% (546) de los 672 encuestados respondió la pregunta sobre el número de horas de trabajo promedio en el día en el año 2014. El 1,8\% trabajaba cuatro horas o menos al día, el 54,9\% reportó trabajar entre cinco y ocho horas en el día, siendo ocho horas el de mayor porcentaje $(48,4 \%)$; el $34,4 \%$ de nueve a doce horas al día, el 6,6\% de trece a dieciséis horas al día y el 2,2\% de dieciocho a veinticuatro horas al día. La media de horas trabajadas al día es de 9,3.

\section{Ingresos en salarios mínimos legales men-} suales vigentes. La tabla 4 presenta el nivel de ingresos según la formación académica; se observa que la mayoría del personal auxiliar y técnico/tecnólogo ganaba entre uno y tres salarios mínimos legales mensuales (SMLMV). En el personal con nivel profesional el mayor porcentaje $(53,8 \%)$ ganaba cuatro o más SMLMV, el 19,0\% ganaba tres salarios mínimos o menos. Entre las personas que tienen diplomado, especialización y/o maestría, el mayor porcentaje tenía ingresos entre cuatro o más SMLMV.

El 62,6\% de los encuestados afirmó que en los contratos laborales de los últimos tres años se estableció la fecha de pago de su salario, el $20,5 \%$ dijo que no y el $16,8 \%$ no recuerda o no respondió la pregunta. La pregunta sobre oportunidad en el pago del salario la contestaron el 83,5\% (561) de los 672 encuestados. De estos, al 63,6\% el pago se le hizo en las fechas estipuladas, al 18,7\% se le tardó hasta un mes, al 8,2\% hasta dos meses, al 3,2\% hasta tres meses, al 3,0\% cuatro meses, al $1,2 \%$ cinco meses, al $0,9 \%$ seis meses y al $1,1 \%$ restante entre 7 y 31 meses.

En cuanto al pago de prestaciones sociales, el 47,9\% recibió prima de vacaciones, el 20,8 bonificaciones, el 14,3\%, aguinaldo, el 9,7\% 


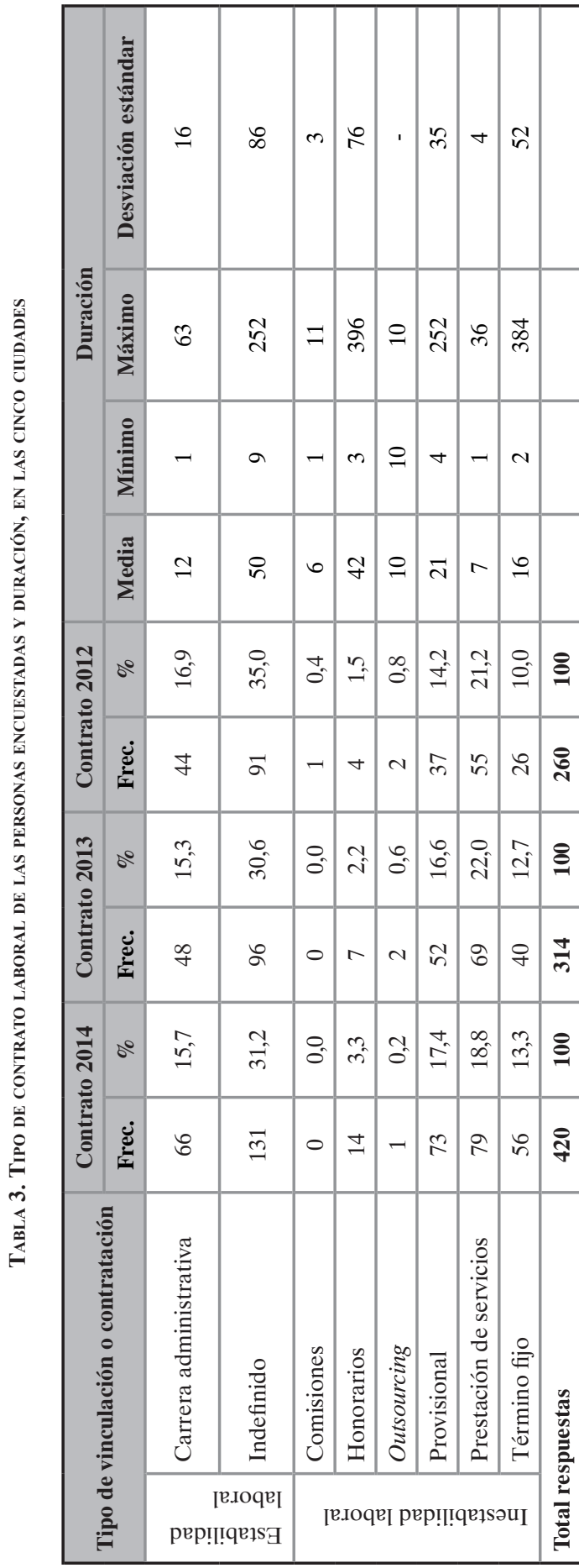

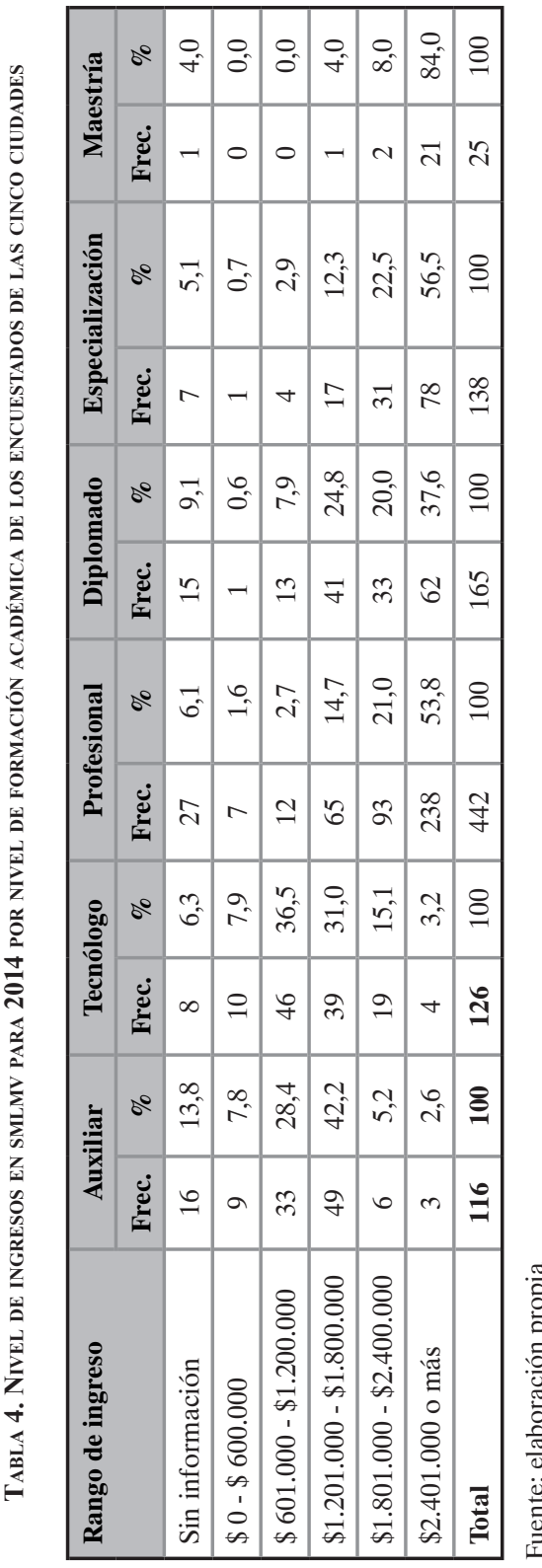


Gloria Molina-Marim • Tatiana Oquendo-Lozano • Sergio Rodriguez-Garzón • Nilton Montoya-

Gómez • Celmira Vesga-Gómez • Nancy Lagos-Campos • Rita Almanza-Payares • Martha

Chavarro-Medina • Eloina Goenaga-Jiménez • Gladys Arboleda-Posada

Tabla 5. Afiliación a la Seguridad social de los encuestados de las Cinco CiUdades 2012-2014

\begin{tabular}{|l|c|c|c|c|c|c|}
\hline \multirow{2}{*}{ Afiliación a seguridad social } & \multicolumn{2}{|c|}{2012} & \multicolumn{2}{c|}{2013} & \multicolumn{2}{c|}{2014} \\
\cline { 2 - 7 } & Frecuencia & \% & Frecuencia & \% & Frecuencia & \% \\
\hline Salud & 259 & 38,5 & 314 & 46,7 & 420 & 62,5 \\
\hline Pensiones & 255 & 37,9 & 312 & 46,4 & 417 & 62,1 \\
\hline Riesgos laborales & 249 & 37,1 & 306 & 45,5 & 409 & 60,9 \\
\hline
\end{tabular}

Fuente: elaboración propia

auxilios y el 35,3\% no recibió ningún tipo de prestación social.

Afiliación a seguridad social. La tabla 5 presenta la afiliación a salud, pensiones y riesgos profesionales. Esta información muestra un incremento entre el 2012 y el 2014, pasando en salud de $38,5 \%$ (2012) a $62,5 \%$ (2014).

Gestión del talento humano. Del total de encuestados (672), el 78,1\% respondió que la institución donde trabajaba tiene un departamento o persona encargada de la gestión del talento humano, el 3,7\% dice no tener esta estructura administrativa en la institución y el 18,2\% no sabía o no respondió la pregunta. En la pregunta sobre si la institución tenía definidas las competencias requeridas para el cargo que desempeña, el 72,5\% respondió de forma afirmativa, el 6,3\% dijo que no y el 21,2\% no sabía o no respondió.

Criterios de selección de personal. En una escala de uno a cien (siendo uno el menos importante y cien el más importante), los encuestados le dieron un puntaje de 68,4 a la importancia que le dan los directivos a la formación académica para la selección del personal, 56,5 a los años de experiencia, 48,1 a aprobar examen de conocimiento, 46,6 a aprobar pruebas psicotécnicas, 42,6 a realizar entrevista, 35,5 a la amistad con el directivo, 35,3 al respaldo de un grupo político y 20,1 a otros. Las personas que seleccionaron la opción de otros, mencionaron los siguientes aspectos como importantes para los directivos al realizar la selección del personal: a) presentación personal (apariencia física, buena actitud e interés en el trabajo; veinte personas lo mencionaron); b) disponibilidad laboral (tiempo) o disposición del trabajador (nueve personas); c) necesidad del servicio o de la institución (existencia o disponibilidad del cargo y urgencia de cubrirlo; nueve personas); d) regionalismo (ser de la región donde se desarrollara el contrato, lugar de procedencia o residencia; cuatro personas); e) la edad (tres personas); f) el haber realizado prácticas académicas en la institución, profesionales propositivos para la institución, las normas y el azar; g) capacidad de atención al público, liderazgo en los procesos, trabajo en equipo, afinidad por el programa, compromiso, institución donde estudió, formación académica continua, manejo de sistemas, estabilidad en empleos previos, referencia de otro empleo, trayectoria y evaluación del desempeño laboral. En cuanto a la amistad con algún directivo y/o políticos, 21 personas mencionaron aspectos como: referencias, recomendaciones, relación familiares-directivos, intereses personales, amistad con alguien de la empresa y amistades y respaldo de un grupo político con pago de cuota política.

Capacitación. En cuanto a capacitación en asuntos de salud pública, de los 672 encuestados, el 44,6\% afirmó que la institución cuenta con este plan, el $18,8 \%$ sostuvo que no y el $36,6 \%$ no sabía o no respondió. El 29\% del total de los encuestados manifestó haberse beneficiado de programas de capacitación en asuntos de salud pública ofrecidos por la 


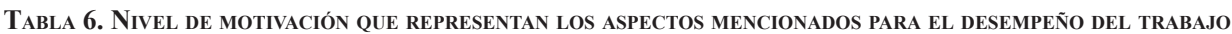
SEGÚN EL TIPO DE INSTITUCIÓN EN LAS CINCO CIUDADES

\begin{tabular}{|l|c|c|c|}
\hline \multirow{2}{*}{ Motivación } & \multicolumn{3}{|c|}{ Tipo de institución } \\
\cline { 2 - 4 } & Mixta & Privada & Pública \\
\hline Salario & 77,3 & 80,0 & 83,6 \\
\hline Reconocimiento & 77,3 & 73,1 & 70,8 \\
\hline Ambiente de trabajo & 77,3 & 78,9 & 80,3 \\
\hline Importancia del cargo & 76,0 & 71,3 & 74,6 \\
\hline Obtención de bonificaciones & 60,0 & 63,0 & 59,7 \\
\hline Posibilidad de acenso y promoción institucional & 74,7 & 68,7 & 63,7 \\
\hline
\end{tabular}

Fuente: elaboración propia

institución, mientras el 56,4\% no se había beneficiado y el 14,6\% no respondió. En el $15,8 \%$ esta capacitación se realizó dentro de la jornada laboral, en $9,8 \%$ algunas veces, en $2,8 \%$ en ningún caso y $71,6 \%$ no respondió la pregunta. El 26,6\% realizó cursos de capacitación por sus propios medios, el 57,3\% no lo hizo y el $16,1 \%$ no respondió la pregunta.

Evaluación. Del total de encuestados, el $67,1 \%$ afirmó que en la institución existían mecanismos periódicos de evaluación del desempeño, el 10,4\% manifestó que no tenían y el 22,5\% no sabía o no respondió la pregunta. En cuanto a la periodicidad de esta evaluación, para el $6,4 \%$ era mensual, para el $38,4 \%$ semestral, para el $11,2 \%$ trimestral, para el $9,5 \%$ anual y el $34,5 \%$ no respondió la pregunta. El 58,5\% de los encuestados respondió que siempre o casi siempre se daban a conocer los resultados de la evaluación realizada, el 3,4\% sostuvo que algunas veces, el 1,8\% nunca y el $36,3 \%$ no sabía o no respondió.

A la pregunta de si los resultados de la evaluación generaban plan de mejoramiento, el $51,6 \%$ respondió que siempre o casi siempre, el 7,0\% que algunas veces, el 3,0\% nunca y el $38,4 \%$ no sabía o no respondió. Si como resultado de la evaluación se generaba alguna sanción, el 27,7\% respondió que nunca, el $13,4 \%$ dijo que algunas veces, el $4,0 \%$ dijo que siempre o casi siempre y el 54,9\% no sabía o no respondió. A la pregunta de si a partir de la evaluación realizada se canceló el contrato laboral, el 31,0\% dijo que nunca, el 8,3\% algunas veces, el 1,7\% siempre o casi siempre y el 58,9\% no sabía o no respondió. Si a partir de la evaluación se dieron incentivos, el 36,3\% dijo que nunca, el 9,4\% manifestó que algunas veces, el 4,2\% siempre o casi siempre y el 50,1\% no sabía o no respondió.

Motivación. La tabla 6 muestra, en una escala de uno a cien, el nivel de motivación que representan algunos criterios para el desempeño laboral de los encuestados, según el tipo de institución. En primer lugar se ubica el salario, tanto en las instituciones públicas como en las privadas, seguido del ambiente laboral, la importancia del cargo, el reconocimiento, la posibilidad de acenso y promoción institucional y la obtención de bonificaciones.

\section{Clima organizacional, satisfacción y recono-} cimientos. En relación con la infraestructura del sitio de trabajo, el 52,7\% la calificó como muy adecuada o adecuada, el 15,2\% como regular y el $32,4 \%$ como inadecuada o muy inadecuada; el 14,7\% no respondió la pregunta.

La comunicación con sus jefes inmediatos fue calificada como buena por el $47,9 \%$ de los encuestados, excelente por el $27,4 \%$, regular 
Gloria Molina-Marím • Tatiana Oquendo-Lozano • Sergio Rodriguez-Garzón • Nilton Montoya-

Gómez • Celmira Vesga-Gómez • Nancy Lagos-Campos • Rita Almanza-Payares • Martha

Chavarro-Medina • Eloina Goenaga-Jiménez • Gladys Arboleda-Posada

por el 8,6\%, mala o deficiente por el 1,6\%, en tanto que el $14,7 \%$ no respondió la pregunta. La comunicación con sus compañeros de trabajo fue calificada como buena por el $49,4 \%$, excelente por el $30,7 \%$, regular por el $4,2 \%$, mala o deficiente por el $0,9 \%$, y el $15 \%$ no respondió la pregunta.

En cuanto a la socialización que los superiores hacen de los logros y avances de su área de trabajo y/o de la institución con el trabajador, el 54,0\% afirmó que se hacía siempre o casi siempre, el 18,6\% ocasionalmente, el 11,9\% casi nunca o nunca y el $15,5 \%$ no respondió.

Con relación a la capacidad del personal para resolver las dificultades sin recurrir a sus superiores, el 61,5\% dijo que siempre o casi siempre existía esa capacidad, el 19,6\% que ocasionalmente, el 3,9\% que nunca o casi nunca y el 15,0\% no respondió la pregunta.

Satisfacción y reconocimiento laboral. Respecto al nivel de satisfacción con la labor desempeñada en la institución, el 77,5\% respondió estar muy satisfecho o satisfecho, el 5,5\%, medianamente satisfecho, el 1,6\% muy insatisfecho o insatisfecho y el $15,3 \%$ restante no respondió esta pregunta. Solo el 23,7\% afirmó haber tenido algún tipo de reconocimiento por su trabajo, el 61,3\% no ha tenido ningún reconocimiento y el $15,0 \%$ no respondió esta pregunta.

La idoneidad en salud pública se evaluó calificando la coherencia entre la formación académica, el cargo y las funciones. Se dio un valor de cero a aquellos en los cuales no había ninguna coherencia entre estos tres aspectos, uno a aquellos que tenían algo de coherencia y dos en los casos en los cuales la formación, el cargo y las funciones eran totalmente coherentes. Luego se exploró la asociación entre la idoneidad con el cargo y doce variables independientes (con los test U de Mann-Whitney y Chi cuadrado de
Pearson), edad, formación académica, tipo de institución, evaluación del desempeño, existencia de un departamento de talento humano, criterios de selección para el cargo como años de experiencia, respaldo político, amistad con los directivos, aprobar pruebas psicotécnicas, aprobar examen de conocimiento, realizar entrevista, capacitación por parte de la institución y por sus propios recursos. Se encontró que las personas clasificadas como idóneas para el cargo asignaron mayor importancia, en promedio, en la selección del personal al ítem "Aprobación del examen de conocimientos", diferencia estadísticamente significativa (Z:-2.33, valor p: 0,02$)$. De igual manera, las personas con mayor idoneidad manifestaron que en la institución donde laboran actualmente se realizaba proceso de evaluación de desempeño (Chi: 24,515, valor p: 0,0000a).

\section{Discusión}

\section{Formación académica, capacitación y desempeño}

La gobernanza y acción en salud pública requiere, entre otros aspectos, personal idóneo con el fin de asegurar el logro de los objetivos y metas en este campo. Los resultados del estudio muestran que la mayoría del talento humano vinculado a servicios y programas en salud pública tenía formación en medicina, enfermería y odontología; muy pocos profesionales tenían maestría en salud pública y/o posgrado en temas afines a esta. También había personal de una amplia gama de áreas de formación tanto de pregrado como de posgrado. Sin embargo, tomando en cuenta los criterios de selección aplicados en los procesos de contratación, los cuales incluyeron amistades políticas, esta diversidad disciplinar no obedece a un plan estratégico o a un enfoque de salud pública. Esta diversidad interdisciplinar no planeada, 
más bien improvisada, podría relacionarse con el clientelismo político y los bajos logros en salud pública en el contexto del sGsss. Este aspecto llama a la necesidad de una coherencia entre la formación disciplinar en salud pública, los cargos y las funciones. Los resultados también muestran una baja cobertura de los programas de capacitación en salud pública ofrecidos por las instituciones, y solo el 26,6\% de los encuestados había realizado capacitación en este campo por sus propios medios. Adicionalmente, aunque los procesos de evaluación se dan en la mayor parte de instituciones con alguna periodicidad, estos no necesariamente conllevaron planes de mejoramiento o incentivos, entre otros.

En este sentido, Herrera (7) afirma que la gestión del talento humano en salud también implica la alineación de los aportes de cada persona con los propósitos institucionales, asunto que involucra conceptos como competencia, idoneidad y capacidades. Este mismo autor ha señalado tres dimensiones del concepto de competencia: a) la competencia como capacidad real, es decir, tangible, observable y demostrable; b) la competencia implica las esferas de conocimientos (saber), actitudes (saber ser) y aptitudes (saber hacer); c) la competencia conlleva una relación de alineamiento entre el desempeño individual y los propósitos de la organización. También García et al. (16) definen competencia como "el conjunto de saberes, cualidades y comportamientos puestos en juego para resolver situaciones concretas de trabajo", lo que está en sintonía con la Unesco que define competencia como "el conjunto de comportamientos socio-afectivos y habilidades cognoscitivas, psicológicas, sensoriales y motoras que permiten llevar a cabo adecuadamente un desempeño, una función, una actividad o una tarea". En salud, Irigoin y Vargas (17) entienden por competencias "una combinación integrada de conocimientos, habilidades y actitudes conducentes a un desempeño adecuado y oportuno en diversos contextos", si estas competencias están en coherencia con las funciones del cargo. En Colombia, la Academia Nacional de Medicina, citada por Puerto (18), define las competencias como: "actuaciones idóneas de un profesional de la salud en su desempeño en un contexto social determinado. Esas capacidades o competencias se configuran mediante la conjunción sistémica de conocimientos, destrezas, aptitudes y actitudes, que expresan valores personales y sociales". Este concepto fue retomado por la legislación colombiana en la Ley 1164 del 2007, donde "competencia es una actuación idónea que emerge en una tarea concreta, en un contexto determinado. Esta actuación se logra con la adquisición y desarrollo de conocimientos, destrezas, habilidades, aptitudes y actitudes que se expresan en el ser, saber, el hacer y el saber hacer" (6). Los resultados de este estudio sugieren una carencia en los procesos de gestión del talento humano.

Un estudio realizado en México concluye que la capacitación ha permitido fortalecer en el personal de salud las competencias para la promoción de la salud y el desarrollo de procesos de trabajo más sostenibles en la comunidad, impulsando una gestión concertada y participativa (19).

\section{Condiciones laborales}

Los hallazgos del estudio muestran que las condiciones laborales del personal que trabajaba en programas de salud pública eran problemáticas, dado que un alto porcentaje (53,0\% en el 2014) del personal tenía un tipo de contrato que implicaba inestabilidad laboral, lo que conllevaba alta rotación de personal. El porcentaje de personas contratadas a término fijo o de carrera administrativa disminuyó entre el 2012 y el 2014. El 9\% había trabajado alguna vez sin contrato; a algunos les tomó varios meses conseguir el empleo (media cinco 
Gloria Molina-Marím • Tatiana Oquendo-Lozano • Sergio Rodriguez-Garzón • Nilton Montoya-

Gómez • Celmira Vesga-Gómez • Nancy Lagos-Campos • Rita Almanza-Payares • Martha

Chavarro-Medina • Eloina Goenaga-Jiménez • Gladys Arboleda-Posada

meses); el 18,5\% de las personas trabajaba en dos o más instituciones simultáneamente; se trabajaba en promedio nueve o más horas al día, es decir, 54 horas por semana; el 36,4\% había tenido demoras en el pago de los salarios; el 35,0\% no recibía ningún tipo de prestaciones sociales; de 38,0 a 40,0\% no tenía afiliación a salud, pensiones o riesgos profesiones en el 2014 y menos aún en el 2013 y 2012.

Otros estudios realizados señalan que en Colombia, respondiendo a las directrices de los organismos multilaterales, se generaron reformas laborales a la par con el sGsss que incluyeron mecanismos de flexibilidad laboral, los cuales han conducido a un deterioro progresivo de las condiciones laborales del personal de salud $(20,21)$. Un estudio realizado en un hospital público de Bogotá muestra que las limitaciones de las políticas y la gestión del recurso humano (contratación, salario, ascensos, salud ocupacional, capacitación, tiempo libre, etc.) son el factor de mayor influencia negativa en el bienestar del personal de enfermería, principalmente en los cooperados y profesionales (22). En este mismo sentido, Flórez et al. (23) afirman que en Colombia la reforma al sistema de salud (Ley 100 de 1993) y las reformas laborales llevadas a cabo paralelamente han contribuido al deterioro de las condiciones laborales del recurso humano en salud.

En el ámbito internacional, Nigenda et al. (1) encontraron una discordancia en la relación entre la formación y la capacitación del personal y su desempeño laboral, deficiente planificación, regulación y condiciones laborales, lo que impacta negativamente en la atención en salud. La deficiencia en la planificación de los recursos humanos, la calidad del entrenamiento y la práctica $(1,24)$ se relacionan con la calidad de los servicios de salud y el desempeño de los profesionales. Con las reformas laborales y de los sistemas de salud se generó deterioro en las condiciones laborales, la formación y la satisfacción del personal (4). En efecto, condiciones laborales desfavorables y bajos salarios conducen al pluriempleo, esto es, a realizar varias actividades laborales en diversas instituciones con el fin de mejorar los ingresos (25), lo que en este estudio se evidencia en que uno de cada cinco de los encuestados trabaja en dos o más instituciones.

Otro estudio señaló que la articulación entre el sector salud y el sector educación permitiría mejorar la calidad de los servicios y orientar la oferta educativa a las necesidades (26).

Otras investigaciones han señalado el impacto que las reformas a los sistemas de salud generaron sobre el talento humano; impacto que si bien se presenta de forma diferente en cada país, sus efectos en los profesionales y en los resultados de la prestación de los servicios constituyen un fenómeno generalizado $(27,28)$. Un estudio en Canadá reportó mayor carga de trabajo e insatisfacción laboral ligada a las reformas realizadas (29); en Chile se ha afectado la capacidad de gestión y el desempeño de los hospitales (30). En México, Ortiz et al. (31) plantean que la calidad de vida laboral y la satisfacción con el empleo se vinculan con la sobrecarga de trabajo, escasez de personal y de recursos para trabajar, los turnos de trabajo, la ambigüedad en las funciones y tareas, la falta de autonomía y autoridad para tomar decisiones, la burocracia y los rápidos cambios tecnológicos.

En Argentina la reforma del sector salud ha producido cambios en la organización de los servicios. Algunos de estos repercutieron fuertemente sobre las prácticas profesionales y las condiciones laborales, agregando nuevos problemas a los ya existentes. Algunas de las características actuales de este campo son la desigual distribución geográfica de profesionales, la estructura del equipo de salud es distorsionada, hay un déficit marcado de per- 
sonal de enfermería, persisten los modelos de formación tradicionales, existe una creciente especialización de los profesionales, un sistema de residencias subdimensionado y sesgado, falta estímulo a la formación en carreras estratégicas y al trabajo en áreas críticas, y hay flexibilidad creciente de las formas de contratación y precariedad laboral en todas las categorías (32).

La globalización es un proceso mundial irreversible de interacción sin barreras desde los individuos, marcado por las tecnologías de la información, que está acentuando la migración de los recursos humanos en salud. En esta migración intervienen factores expulsores: bajos sueldos, nula motivación, condiciones de trabajo peligrosas y adversas, etc. Y factores atrayentes: superiores sueldos, mejores condiciones de vida y laborales, etc. (33).

Las reformas de salud han implicado desafíos en la adecuación de la formación del talento humano, lo cual en los diferentes países se ha quedado rezagado (34). Se plantea el papel que los recursos humanos en la salud deberían desempeñar en las próximas etapas de la reforma del sistema de salud mexicano, a través de las siguientes dimensiones: orientación de la formación, instituciones responsables de esta formación, formas de vincular a los trabajadores a las instituciones y las formas en las que los trabajadores de la salud deben responder a los cambios en marcha. Los cambios requeridos deben estar basados en una propuesta de planificación estratégica sustentada en acuerdos institucionales que definan objetivos comunes, así como procedimientos claros para cumplir las metas (35).

\section{Conclusiones}

- La mayor parte del personal vinculado a los programas de salud pública tiene formación en medicina, enfermería y odontología, entre otras. Sin embargo, hay personal que pertenece a una amplia gama de áreas de formación tanto de pregrado como de posgrado, sin entrenamiento específico en salud pública, dejando un vacío entre el perfil profesional y lo requerido para desempeñar las funciones del cargo.

- Las condiciones laborales del personal de los programas de salud pública son poblemáticas, dado que más de la mitad (53\% para el 2014) estaba vinculado mediante tipos de contratación que conllevan inestabilidad laboral y alta rotación de personal; el personal vinculado a término fijo o de carrera administrativa disminuyó entre el 2012 y el 2014; a algunos les tomó varios meses conseguir el empleo (media cinco meses); el 18,5\% de las personas trabajaba en dos o más instituciones simultáneamente; jornada laboral mayor a la estipulada legalmente (promedio diez o más horas al día); para un $36,4 \%$ se daban demoras en el pago de los salarios; el 35\% no recibía ningún tipo de prestaciones sociales, un porcentaje entre 38 y $40 \%$ no tenía afiliación a salud, pensiones o riesgos profesiones en el 2014 y menos aún en el 2013 y 2012.

- Había poca cobertura con los programas capacitación en salud pública ofrecidos por las instituciones para las cuales trabajan; igualmente, solo el 26,6\% había realizado capacitación por sus propios medios.

- A pesar de que en el 67\% de los encuestados manifestó que en la institución donde laboraba se realizaban procesos periódicos de evaluación de personal, en solo la mitad se daba a conocer esta evaluación y se realizaban planes de mejoramiento.

- El salario, el reconocimiento y el ambiente laboral fueron ubicados como 
Gloria Molina-Marím • Tatiana Oquendo-Lozano • Sergio Rodríguez-Garzón • Nilton Montoya-

Gómez • Celmira Vesga-Gómez • Nancy Lagos-Campos • Rita Almanza-Payares • Martha

Chavarro-Medina • Eloina Goenaga-Jiménez • Gladys Arboleda-Posada

los aspectos que más motivaban a las personas encuestadas.

- La mayor parte de los encuestados calificaron como buena y excelente la comunicación con el jefe y los compañeros de trabajo.

- Un bajo porcentaje (23\%) afirmó haber tenido algún tipo de reconocimiento por su trabajo.

- La idoneidad en salud pública de las personas encuestadas se asocia con la aprobación del examen de conocimiento durante el proceso de selección para el cargo y con la existencia de procesos de evaluación del desempeño en las instituciones donde los encuestados trabajaban.

\section{Agradecimientos}

En primer lugar, se agradece a las instituciones de salud y a las personas seleccionadas que contribuyeron con su información y sin lo cual este estudio no se hubiera podido llevar a cabo. También agradecemos a Colciencias (proyecto Decisiones en la política y la gestión en salud pública, contrato $\mathrm{n}^{\circ} 111554531444$ ), y al CODI — estrategia de sostenibilidad 20132014 — de la Universidad de Antioquia, por el apoyo con la financiación.

\section{Referencias}

1. Nigenda G, Ruiz JA, González LM, Wirtz V, González MC, Aguilar ME y Bejarano R. Formación, empleo y regulación de los recursos humanos para la salud. Bases para su planeación estratégica. México: Instituto Nacional de Salud Pública y Cendex; 2010.

2. Organización Mundial de la Salud. Human Resources for Health. Overcoming the crisis. Washington D.C.: Library of Congress; 2004.

3. Costa IA, Palucci MH. Sistemas de salud en búsqueda de excelencia: los recursos humanos en foco.
Revista Latinoamericana de Enfermería. 2006; 14 (3): 307-8.

4. Organización Panamericana de la Salud, Ontario y Canadá. Llamado a la acción de Toronto. Hacia una década de Recursos Humanos en Salud para las Américas. Toronto: Reunión regional de los observatorios de recursos humanos en salud. 2005. Disponible en: http://www.observatoriorh.org/sites/ default/files/webfiles/fulltext/ors_desafios_toronto_2005.pdf

5. Organización Mundial de la Salud. Colaboremos por la salud. Informe sobre la salud en el mundo. Francia: oms; 2006.

6. Colombia, Congreso de la República. Ley 1164 de 2007, Art. 1.

7. Herrera Á. Modelo aplicado de gestión humana basada en competencias - Manual didáctico. Cali: Sello Editorial Javeriano; 2007.

8. Chiavenato I. Administración de Recursos Humanos: El capital humano de las organizaciones, novena edición. México: McGraw-Hill, Interamericana Editores; 2011.

9. Novick M, Rosales C. Desafíos de la gestión de los recursos humanos en salud: 2005-2015. Washington D.C.: ops; 2006.

10. Restrepo J, Lopera J, Rodríguez S. Integración vertical en el sistema de salud colombiano: aproximaciones empíricas y análisis de doble marginalización. Borradores del CIE. 2005; 15.

11. Colombia, Defensoría del Pueblo. Autonomía médica y su relación con la prestación de los servicios de salud. Bogotá: Defensoría del Pueblo; 2007.

12. Molina G, Ramírez A, Muñoz I, editores. Dilemas en las decisiones en la atención en salud. Ética, derechos y deberes constitucionales frente a la rentabilidad financiera en el sistema de salud colombiano, segunda edición. Bogotá: Editorial La Carreta; 2011.

13. González LE. Catorce EPS serían multadas por hacer 'pacto' para negar servicios. Periódico el Tiempo. 2011 abril; 23: 1.

14. Montero J. Estadística descriptiva. Madrid: Editora de Producción; 2007.

15. Campos M. Más de 600 preguntas cortas de bioestadísticas y sus respuestas. Murcia: Universidad de Murcia; 2007.

16. Irigoin M, Vargas F. Competencia laboral: Manual de conceptos, métodos y aplicaciones zen el sector salud. Montevideo: ops; 2002.

17. García JA, González, JF, Estrada L, Uriega S. Educación médica basada en competencias. Revista Médica del Hospital General de México. 2010; 73 (1): 57-69.

18. Puerto N. Referentes conceptuales y normativos sobre competencias profesionales en salud. Disponible en: http://www.minsalud.gov.co/salud/Documents/Observatorio\%20Talento \%20Humano\%20 en $\% 20$ Salud/3-ReferentesNormativos \%20.pdf

19. Alcalde JE, Molina JF, Castillo LE. Aportes de la capacitación a la promoción de la salud en los Servicios Estatales de Salud: análisis comparativo en ocho estados de México. Revista Salud Pública de México. 2013; 55 (3): 285-94. 
20. Flórez JH, Atehortúa SC, Arenas AC. Las condiciones laborales de los profesionales de la salud a partir de la Ley 100 de 1993: evolución y un estudio de caso para Medellín. Rev. Gerenc Políticas Salud. 2009; 8 (16): 107-31.

21. Cristo P, Caballero R, Atanache R, Bautista W, Mahecha A, Ortiz C, et al. Impacto económico y laboral del Sistema General de Seguridad Social en Salud en el ejercicio profesional de los odontólogos egresados del Colegio Odontológico Colombiano 1979-1991. Ces Odontol. 2005; 18 (2): 29-36.

22. Guerrero J, Pulido G. Trabajo, salud y régimen contractual en personal de enfermería: un enfoque psicosocial. Revista Avances en Enfermería; 2010; 28 (2): 111-22.

23. Flórez, JH, Atehortúa SC y Arenas AC. Las condiciones laborales de los profesionales de la salud a partir de la Ley 100 de 1993: evolución y un estudio de caso para Medellín. Revista Gerencia y Políticas de Salud. 2009; 8: 107-31.

24. Brito PE, Padilla M y Rigoli F. Planificación de recursos humanos y reformas del sector salud. Revista Cubana de Educación Médica Superior. 2012; 16 (4).

25. Organización Panamericana de la Salud (OPs). Estudio comparativo de las condiciones de trabajo y salud de los trabajadores de la salud en: Argentina, Brasil, Costa Rica y Perú. Washington, D.C.: OPS; 2012.

26. Organización Panamericana de la Salud (ops). CurSo virtual FESP: Enfoques, problemas y perspectivas de la formación de los recursos humanos en salud. 2009. Disponible en: http://ops.oer.bvsalud.org/ index.php? $\mathrm{P}=$ FullRecordyID $=47$

27. Brito PE. Impacto de las reformas del sector de la salud sobre los recursos humanos y la gestión laboral. Revista Panameña de Salud Pública. 2000; 8 (2): 43-54.
28. Del Valle AH. Reformas estructurales, mercado de trabajo y protección social en América Latina. Portularia Rev Trab Soc. 2010; 10 (1): 3-21.

29. Isik U, Zeytinoglu, Denton M, Davies S, Baumann A, Blythe J, Boosi L. Associations between Work Intensification, Stress and Job Satisfaction. Relations Industrielles / Industrial Relations. 2007; 62 (2): 201-25.

30. Méndez CA, Torres MC. Hospital management autonomy in Chile: the challenges for human resources in health. Revista Saúde Pública. 2010; 44 (2): $1-5$.

31. Ortiz JA, Arias F. El desgaste profesional (Burnout) y calidad de vida laboral como predictores de la búsqueda de otro trabajo en profesionales de la salud y de la educación en el occidente de México. Revista Ciencia \& Trabajo. 2011; (34): 222-6.

32. Rovere M, Abramzon M. Recursos Humanos en Salud. Bases para una agenda impostergable. IX Jornadas Nacionales de Debate Interdisciplinario en Salud y Población. 2011. Buenos Aires, Argentina [acceso: diciembre del 2014]. Disponible en: http:/www.econ.uba.ar/planfenix/docnews/ III/Politicas\%20de\%20desarrollo. \%20RRHH/ Abramzon.pdf

33. Sotomayor R. Globalización y la responsabilidad de los países en desarrollo: el caso de la migración de los profesionales de salud. Revista Peruana de Medicina Experimental y Salud Pública. 2007; 24 (3): 300-6.

34. Méndez CA. Los recursos humanos de salud en Chile: el desafío pendiente de la reforma. Revista Panamericana de Salud Pública; 26 (3): 276-80.

35. Nigenda G, Magaña-Valladares L, Ortega-Altamirano DV. Recursos Humanos para la Salud en el Contexto de la Reforma Sanitaria en México: Formación Profesional y Mercado Laboral. Gaceta Médica de México. 2013; 149: 555-61. 